\title{
Organic Conductors: Evidence for Correlation Effects in Infrared Properties
}

\author{
Jacobsen, Claus Schelde; Johannsen, Ib; Bechgaard, Klaus
}

Published in:

Physical Review Letters

Link to article, DOI:

10.1103/PhysRevLett.53.194

Publication date:

1984

Document Version

Publisher's PDF, also known as Version of record

Link back to DTU Orbit

Citation (APA):

Jacobsen, C. S., Johannsen, I., \& Bechgaard, K. (1984). Organic Conductors: Evidence for Correlation Effects in Infrared Properties. Physical Review Letters, 53(2), 194-197. https://doi.org/10.1103/PhysRevLett.53.194

\section{General rights}

Copyright and moral rights for the publications made accessible in the public portal are retained by the authors and/or other copyright owners and it is a condition of accessing publications that users recognise and abide by the legal requirements associated with these rights.

- Users may download and print one copy of any publication from the public portal for the purpose of private study or research.

- You may not further distribute the material or use it for any profit-making activity or commercial gain

- You may freely distribute the URL identifying the publication in the public portal 


\title{
Organic Conductors: Evidence for Correlation Effects in Infrared Properties
}

\author{
C. S. Jacobsen and Ib Johannsen \\ Physics Laboratory III, Technical University of Denmark, DK-2800 Lyngby, Denmark \\ and
}

\author{
K. Bechgaard \\ Department of General and Organic Chemistry, H. C. Ørsted Institute, DK-2100 Copenhagen, Denmark
}

(Received 12 December 1983)

\begin{abstract}
The infrared conductivities of four organic conductors with partially filled one-electron bands are compared. The behavior ranges from near Drude type in the best metal to semiconductorlike in the moderate conductor. Electron-molecular-vibration coupling effects of varying degree are seen in all materials. It is suggested that the combined effect of electronelectron interaction and electron-phonon interaction in producing $4 k_{\mathrm{F}}$ charge-density waves is essential for interpreting the results.
\end{abstract}

PACS numbers: $78.30 . \mathrm{Jw}, 71.38 .+\mathrm{i}, 72.80 . \mathrm{Le}$

It is well known that the formal requirement of a partially filled one-electron band in quasi-onedimensional organic conductors ${ }^{1}$ is not sufficient to ensure metallic transport properties. ${ }^{2,3}$ The Peierls and Overhauser instabilities ${ }^{4}$ tend, by creating $2 k_{\mathrm{F}}$ potentials, to destroy the Fermi surface in these materials. However, the electronic structure may, already at high temperatures, be dominated by strong Coulomb interactions, i.e., delocalization of the carriers is too costly in electrostatic energy.

The susceptibility of organic conductors to such correlation effects is believed to arise from (1) narrow electronic bands (widths of order $0.3-1 \mathrm{eV}$ ), (2) low carrier densities $\left[(1-3) \times 10^{21} \mathrm{~cm}^{-3}\right]$, and (3) the linear-chain structure, which makes it difficult for the carriers to avoid each other.

Correlation effects are usually discussed in terms of the extended Hubbard model ${ }^{5}$ :

$$
\begin{aligned}
H=- & \sum_{i, \sigma} t\left(c_{i, \sigma}^{\dagger} c_{i+1, \sigma}+c_{i+1, \sigma}^{\dagger} c_{i, \sigma}\right)+\sum_{i} U n_{i \uparrow} n_{i \downarrow} \\
& +\sum_{i, \sigma, \sigma^{\prime}} V n_{i, \sigma} n_{i+1, \sigma^{\prime}}
\end{aligned}
$$

where $t$ is the overlap integral, $U$ is the on-site and $V$ the nearest-neighbor Coulomb interaction. $c_{i, \sigma}^{\dagger}$ creates an electron of spin $\sigma$ at site $i$, and $n_{i, \sigma}$ $=c_{i, \sigma}^{\dagger} c_{i, \sigma}$ is the occupation number. Recently, Mazumdar and $\mathrm{Bloch}^{3}$ have shown that more distant Coulomb interactions can be neglected and that the effective, screened values of $U$ and $V$ depend strongly on band filling, which is given by the degree of charge transfer, $\rho$. Screening is found to be inefficient for $\rho=1$ (half-filled band), resulting in a Mott insulator. ${ }^{6}$ The screening is most efficient for $\rho=0.7-0.8$, where Coulomb interactions may be negligible compared with the bandwidth, $4 t$. Ap- proaching the quarter-filled band $(\rho \rightarrow 0.5)$, the screening again becomes inefficient.

A generally accepted experimental probe for important correlation effects is the observation of diffuse $\mathrm{x}$-ray scattering at wave vector $4 k_{\mathrm{F}}{ }^{7}$ Two distinct limits of Eq. (1) account for such scattering: With $t \rightarrow 0$, Hubbard ${ }^{5}$ found generalized Wigner lattices with strong $4 k_{\mathrm{F}}$ components in the electron density. A finite electron-phonon coupling renders the Wigner lattice visible in $\mathrm{x}$-ray diffraction through the formation of an accompanying lattice distortion. The effect of a finite $t$ in this model is unclear. The other and perhaps more appropriate limit is $U \rightarrow \infty$ ( $V$ is neglected), where double occupancy of sites is excluded. Then the electrons behave like spinless fermions and the density of states is halved. The conventional Peierls instability, driven by the electron-phonon interaction, will then occur at $4 k_{\mathrm{F}}$ and open a gap in the excitation spectrum. $^{8}$

We have examined the infrared properties of four organic conductors at $T=300 \mathrm{~K}$, where $2 k_{\mathrm{F}}$ scattering is weak or undetectable. They range from good metals to a true Mott insulator and span values of $\rho$ from 0.55 to 1.0. They all satisfy the requirement of a partly filled one-electron band, and have no structural disorder. Thus deviations from the normal metallic Drude absorption may be ascribed to correlation effects combined with electron-phonon interactions, although effects of dimensionality can play an additional role. ${ }^{9}$

Single crystals or assemblies of single crystals were investigated by means of polarized reflectance measurements. Here we present the infrared part of the chain axis conductivity, $\sigma(\omega)$, as obtained from reflectance by Kramers-Kronig analysis using 
suitable extrapolation procedures. ${ }^{10}$

Figure 1 shows $\sigma(\omega)$ for the four double stack conductors: (1) HMTSF-TNAP (hexamethylenetetraselenafulvalene-tetracyanonaphtoquinodimethane), which has a very high dc conductivity $\left[\sigma_{\mathrm{dc}}\right.$ $(300 \mathrm{~K})=2400 \Omega^{-1} \mathrm{~cm}^{-1}, 11 \rho \simeq 0.58^{12}$ ]; (2) TTF-TCNQ (tetrathiafulvalene-tetracyanoquinodimethane) $\left[\sigma_{\mathrm{dc}}(300 \mathrm{~K})=500-1000 \Omega^{-1} \mathrm{~cm}^{-1},{ }^{13}\right.$ $\left.\rho=0.55^{14}\right]$; this spectrum has previously been published $^{15}$; (3) DBTTF-TCNQCl 2 (dibenzo-TTFdichloro-TCNQ), a moderate conductor $\left[\sigma_{\mathrm{dc}}(300\right.$ $\left.\mathrm{K})=40 \Omega^{-1} \mathrm{~cm}^{-1},{ }^{16} \rho=0.56^{17}\right]$; and (4) DBTSF$\mathrm{TCNQF}_{4}$ (dibenzo-TSF-tetrafluoro-TCNQ), a Mott insulator ${ }^{18,19}\left[\sigma_{\mathrm{dc}}(300 \mathrm{~K})=10^{-4} \Omega^{-1} \mathrm{~cm}^{-1}\right.$, $\rho=1$ ]. Infrared data on DBTSF-TCNQF 4 in agreement with ours also has been obtained by Bryden et al. ${ }^{19}$

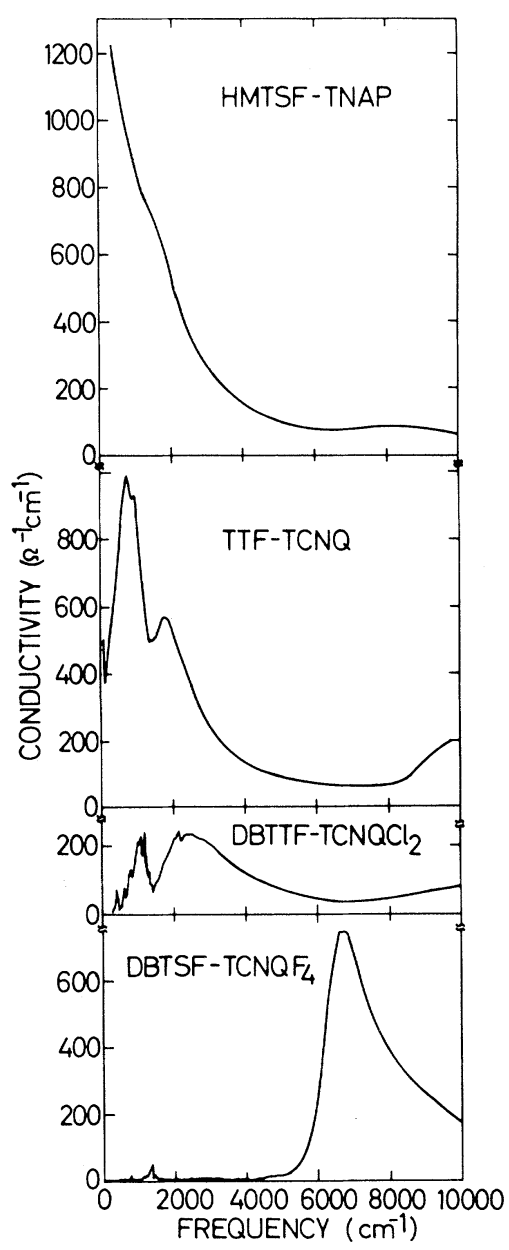

FIG. 1. Infrared chain axis conductivities of the organic conductors HMTSF-TNAP, TTF-TCNQ, DBTTF$\mathrm{TCNQCl}_{2}$, and DBTSF-TCNQF 4 as obtained by dispersion analysis of reflectance data. $T=300 \mathrm{~K}$.
The Lorentzian falloff from $\sigma_{\mathrm{dc}}$ expected from the Drude model ${ }^{10}$ is not seen in any of the materials, although HMTSF-TNAP comes close. Generally, the oscillator strength is centered at the higher frequency for the lower dc conductivity. In addition to the main electronic band, there is evidence for electron-phonon coupling effects in the range of molecular vibrations. Very similar behavior has previously been observed in other groups of materials, most notably in the single stack conductors $\mathrm{TMTCF}_{2} X$ (ditetramethyltetrachalcogenafulvalene- $X){ }^{20}$ However, these have the additional complicating feature of slightly dimerized chains.

Table I summarizes key parameters for the four materials, including $\sigma_{\mathrm{dc}}(300 \mathrm{~K})$, the position of the electronic band, $\omega_{\mathrm{CT}}$, and the total oscillator strength, estimated from the partial sum rule, ${ }^{10}$ $\omega_{p}^{2}=(2 / \pi) \int \epsilon_{2}(\omega) \omega d \omega$ (integrated over all intraband absorption). For noninteracting carriers in a tight-binding band of width $4 t$, this strength in the random-phase approximation ${ }^{10}$ is given by

$$
\omega_{p 0}^{2}=4 t a^{2} e^{2} \sin (\rho \pi / 2) / \pi \epsilon_{0} \hbar^{2} V_{m} .
$$

Here $a$ is the lattice constant in the chain direction, and $V_{m}$ is the volume corresponding to $\rho$ carriers. $4 t$ calculated from this equation with the measured $\omega_{p}^{2}$ is a measure of the oscillator strength with the effect of carrier density roughly eliminated, and is given in the last column of Table I. In general, $4 t$ will underestimate the bandwidth, because short-range correlations tend to suppress $\omega_{p}^{2}$ below $\omega_{p 0} \cdot{ }^{21,22}$ This is most directly demonstrated in the case of DBTTF-TCNQCl ${ }_{2}$, where $4 t=0.22 \mathrm{eV}$ seems rather low compared with estimates based on intermolecular distances. ${ }^{23}$

A crude analysis may be carried out for DBTSF$\mathrm{TCNQF}_{4}$. The half-filled band has been discussed by $\mathrm{Lyo}^{24}$ in the extended Hubbard model. The absorption is dominated by a charge-transfer band corresponding to the creation of a doubly occupied

TABLE I. Conductivities and infrared parameters. $4 t=\pi \omega_{p}^{2} \epsilon_{0} \hbar V_{m} / a^{2} e^{2} \sin (\pi \rho / 2)$.

\begin{tabular}{lcrrr}
\hline \multicolumn{1}{c}{ Material } & $\begin{array}{c}\sigma_{\mathrm{dc}} \\
(\Omega \mathrm{cm})^{-1}\end{array}$ & $\begin{array}{c}\omega_{\mathrm{CT}} \\
\left(\mathrm{cm}^{-1}\right)\end{array}$ & $\begin{array}{c}\omega_{p}^{2} \\
\left(10^{7} \mathrm{~cm}^{-2}\right)\end{array}$ & $\begin{array}{c}4 t \\
(\mathrm{eV})\end{array}$ \\
\hline HMTSF-TNAP & 2400 & $<300$ & 10.3 & 0.68 \\
TTF-TCNQ & 700 & 800 & 9.1 & 0.46 \\
DBTTF-TCNQCl & 40 & 2100 & 3.1 & 0.22 \\
DBTSF-TCNQF $_{4}$ & $10^{-4}$ & 6700 & 7.7 & 0.40 \\
\hline \hline
\end{tabular}


site. Lyo finds an asymmetric peak in $\sigma(\omega)$ with the onset of absorption at $\sim U-4 t$. This is indeed what is observed experimentally (Fig. 1), and we may estimate $U-4 t \simeq 0.7 \mathrm{eV}$. If we assume that the suppression of $\omega_{p}^{2}$ follows that of the isolated Hubbard dimer ${ }^{25} \omega_{p}^{2} / \omega_{p 0}^{2}=\left[1+(U / 4 t)^{2}\right]^{-1 / 2}$ (correct for $U / 4 t \rightarrow 0$ and $\left.U / 4 t>>1^{24}\right)$, we find that $\omega_{p}^{2} / \omega_{p 0}^{2}=0.5, U=1.4 \mathrm{eV}$ and $4 t=0.7 \mathrm{eV}$. Such a bandwidth appears reasonable for a material containing tetraselenafulvalene. ${ }^{20}$

We now concentrate on the three incommensurate organic conductors with $0.5<\rho<1.0$. In the simplest approach one may attempt to model the non-Drude behavior in terms of Eq. (1) in a rigid lattice. For $V=0$ Maldague $^{21}$ found that nearly all the oscillator strength is at low frequencies. Absorption corresponding to $U$ is weak and vanishes rapidly with decreasing $\rho$. The weak band near $8000-10000 \mathrm{~cm}^{-1}$ in these materials could be related to $U,{ }^{26}$ but is more likely attributed to intramolecular excitations. ${ }^{27}$

Mazumdar and Soos ${ }^{28}$ conclude from finite-chain calculations that appreciable oscillator strength may appear near $V$. Thus the optical absorption is assigned to a charge-transfer band, the position of which is determined by the effective nearestneighbor Coulomb interaction. Metallic transport properties may persist, since no gap is expected in the excitation spectrum. ${ }^{28}$ On the basis of our data we cannot exclude that such an effect plays a role; however, it cannot in itself account for the behavior of DBTTF-TCNQCl ${ }_{2}$.

The dc transport properties of DBTTF-TCNQCl 2 are those of a semiconductor with a gap of $\simeq 0.13$ $\mathrm{eV}\left(\sim 1100 \mathrm{~cm}^{-1}\right) .^{16}$ Diffuse $\mathrm{x}$-ray scattering experiments ${ }^{17}$ yield strong $4 k_{\mathrm{F}}$ scattering with fairly one-dimensional character from 300 to $180 \mathrm{~K}$ followed by a three-dimensional ordered phase below $180 \mathrm{~K}$. The infrared absorption shows no significant temperature dependence. Finally, a threedimensional $2 k_{\mathrm{F}}$ distortion develops near $38 \mathrm{~K}$.

With this in mind we suggest that $\sigma(\omega)$ may be described as arising from single-particle transitions across a gap of order $1100 \mathrm{~cm}^{-1}$. The gap is likely due to locking of the electrons in $4 k_{\mathrm{F}}$-chargedensity waves (CDW's), and is most easily rationalized as a combined effect of electron-electron and electron-phonon interactions in the $U \rightarrow \infty$ picture described above. The CDW's in turn couple to the fully symmetric molecular vibration modes and give rise to a strong infrared absorption bands near the mode frequencies (the most important carbon skeleton modes are in the $1000-1400-\mathrm{cm}^{-1}$ range). The analogous effect in the ordinary Peierls $2 k_{\mathrm{F}^{-}}$ distorted system has been extensively discussed by Rice and co-workers. ${ }^{29,30}$ An important aspect is that the coupling to the vibrational modes may significantly stabilize the CDW's (i.e., contribute to the gap). ${ }^{31}$

The incommensurate CDW discussed here may give a finite dc conductivity, since its energy is independent of its phase. However, if CDW's exist on both chains, such a contribution is inhibited by the Coulomb interaction between the oppositely charged density waves. This interaction eventually makes them order three dimensionally.

We may now attempt to apply the same ideas to TTF-TCNQ. The band filling of the two materials is almost the same, but $4 t$ as determined from the oscillator strength is different (Table I). Although these numbers are lower limits for the bandwidths, details in the molecular overlap ${ }^{23}$ as well as other physical properties ${ }^{16}$ strongly suggest that the acceptor bandwidth, and to some extent the donor bandwidth in TTF-TCNQ, is larger than in DBTTF-TCNQCl 2 . This, of course, reduces the importance of correlation effects, but TTF-TCNQ does show $4 k_{\mathrm{F}}$ scattering at $300 \mathrm{~K},{ }^{14}$ while $2 k_{\mathrm{F}}$ scattering is very weak down to $150 \mathrm{~K}^{32}$ We now have to assume that the $4 k_{\mathrm{F}}-\mathrm{CDW}$ formation predominantly takes place on the TTF chain (which has the smaller bandwidth). In that case the TCNQ chain contributes to the infrared conductivity $\sigma(\omega)$, with a near Drude-like absorption, while the TTF chain with a CDW-induced gap gives rise to the peak in $\sigma(\omega)$ at $800 \mathrm{~cm}^{-1}$ and the indentation near $1300 \mathrm{~cm}^{-1}$; the latter feature is again due to electron-molecular-vibration coupling. The rise in $\sigma(\omega)$ for $\omega \rightarrow 0$ may be attributed to sliding $4 k_{\mathrm{F}}$ CDW's, which in this case experience no barrier.

The last material, HMTSF-TNAP, is rather close to Drude-type behavior, with monotonous $\sigma(\omega)$. This is consistent with a relatively big bandwidth $(W>0.68 \mathrm{eV})$. Also, preliminary diffuse $\mathrm{x}$-ray measurements show only low-temperature $2 k_{\mathrm{F}}$ scattering. ${ }^{12}$

In conclusion the present study supports the idea that the influence of correlation effects in organic conductors depends strongly on band filling and bandwidth. The infrared properties have been linked to the observation of $4 k_{\mathrm{F}}$-charge-density waves, which in at least one of the discussed materials, DBTTF-TCNQCl 2 , give rise to a gap in the excitation spectrum. The results directly demonstrate that the electron-phonon interactions cannot be neglected, whatever is the correct mechanism for the CDW formation.

We acknowledge useful discussions with Dr. J. B. 
Torrance, who partly inspired this work. We are grateful to Dr. Pouget for communicating his results to us prior to publication. This work was partially supported by the Royal Danish Academy of Sciences and Letters through a Niels Bohr Fellowship.

${ }^{1}$ For recent progress, see Proceedings of the International Conference on Synthetic Low Dimensional Conductors and Superconductors [J. Phys. (Paris) 44, Colloq. No. 3 (1983)].

2J. B. Torrance, Acc. Chem. Res. 12, 79 (1979).

${ }^{3}$ S. Mazumdar and A. N. Bloch, Phys. Rev. Lett. 50, 207 (1983).

${ }^{4}$ See, for example, H. Gutfreund, in Chemistry and Physics of One-Dimensional Metals, edited by H. J. Keller (Plenum, New York, 1977), pp. 267-278.

5J. Hubbard, Phys. Rev. B 17, 494 (1978), and references therein.

${ }^{6}$ N. F. Mott, Proc. Phys. Soc. London, Sect. A 62, 416 (1949).

${ }^{7}$ See, for example, V. J. Emery, in Highly Conducting One-Dimensional Solids, edited by J. T. Devreese et al. (Plenum, New York, 1979), p. 247.

8J. Bernasconi, M. J. Rice, W. R. Schneider, and S. Strässler, Phys. Rev. B 12, 1090 (1975).

${ }_{9}^{9}$ P. F. Williams and A. N. Bloch, Phys. Rev. B 10, 1097 (1974).

${ }^{10}$ F. Wooten, Optical Properties of Solids (Academic, New York, 1972).

${ }^{11} \mathrm{~K}$. Bechgaard, C. S. Jacobsen, and N. Hessel Andersen, Solid State Commun. 25, 875 (1978).

12J. P. Pouget et al., unpublished.
${ }^{13}$ M. J. Cohen, L. B. Coleman, A. F. Garito, and A. J. Heeger, Phys. Rev. B 10, 1298 (1974).

${ }^{14}$ S. Kagoshima, T. Ishiguro, and H. Anzai, J. Phys. Soc. Jpn. 41, 2061 (1976).

${ }^{15} \mathrm{C}$. S. Jacobsen, in Quasi-One-Dimensional Conductors, edited by S. Barisic, A. Bjelis, J. R. Cooper, and B. Leontic, Lecture Notes in Physics, Vol. 95 (SpringerVerlag, Berlin-Heidelberg, 1979), p. 223.

${ }^{16}$ C. J. Jacobsen, H. J. Pedersen, K. Mortensen, and K. Bechgaard, J. Phys. C 13, 3411 (1980).

${ }^{17} \mathrm{~K}$. Mortensen, C. S. Jacobsen, A. LindegaardAndersen, and K. Bechgaard, J. Phys. (Paris), Colloq. 44, C3-1349 (1983).

${ }^{18} \mathrm{~K}$. Lerstrup et al., J. Chem. Soc. Chem. Commun. 294 (1983).

${ }^{19}$ W. A. Bryden et al., Phys. Rev. B (to be published).

${ }^{20}$ C. S. Jacobsen, D. B. Tanner, and K. Bechgaard, Phys. Rev. B 28, 7019 (1983).

21P. F. Maldague, Phys. Rev. B 16, 2437 (1977).

22I. Sadakata and E. Hanamura, J. Phys. Soc. Jpn. 34, 882 (1973).

${ }^{23}$ H. Soling, G. Rindorf, and N. Thorup, Acta. Crystallogr., Sect. B 37, 1716 (1981).

${ }^{24}$ S. K. Lyo, Phys. Rev. B 18, 1854 (1978).

${ }^{25}$ M. J. Rice, Solid State Commun. 31, 93 (1979).

26 J. B. Torrance, B. A. Scott, and F. B. Kaufman, Solid State Commun. 17, 1369 (1975).

27J. Tanaka et al., Ann. N.Y. Acad Sci. 313, 256 (1978).

${ }^{28}$ S. Mazumdar and Z. G. Soos, Phys. Rev. B 23, 2810 (1981).

${ }^{29}$ M. J. Rice, Phys. Rev. Lett. 37, 36 (1976).

30M. J. Rice, L. Pietronero, and P. Brüesch, Solid State Commun. 21, 757 (1977).

${ }^{31}$ M. J. Rice, C. B. Duke, and N. O. Lipari, Solid State Commun. 17, 1089 (1975).

${ }^{32}$ R. Comes and G. Shirane, Ref. 7, p. 17. 